\title{
Research of Distributed Data Acquisition System Based on CIM/E
}

\author{
Peng DU ${ }^{1, a}$, Ziming GUO ${ }^{2, b}$, Zhaoqi WEN ${ }^{1}$, Hao ZHANG ${ }^{2}$, Yu QIAO ${ }^{1}$, Hao \\ ZHANG ${ }^{2}$, Wenwen WANG ${ }^{1}$, Jianhua CHEN $^{2}$ \\ ${ }^{1}$ NARI Group Corporation (State Grid Electric Power Research Institute), Beijing 100192, China \\ ${ }^{2}$ State Grid Jibei Electric Power Company Limited, Beijing, 100053, China
}

Keywords: data acquisition; distributing; CIM/E; integrated dispatching; reserve dispatching

Abstract: This paper analyzes the traditional mode of data acquisition, proposed the distributed data acquisition system architecture which is based on the CIM/E, while discussing the management of $\mathrm{CIM} / \mathrm{E}$ files and data sharing under this architecture. The feasibility of the system has been verified by engineering experiment. The extension capability, reliability and usability of this system has been greatly promoted and the system is ready to apply to scenarios of coordinate operation of multiple dispatching center, such as province and region integration, region and county integration, the primary and standby integration.

\section{Introduction}

For constructing a' one strong and three excellent 'modern company, playing the advantages of the operation of the group, integrating power grid operating resources, constructing the new power grid operating system, State Grid launched the 'three sets of five major' system construction. According to the 'three sets of five major' strategic requirements, State Grid will establish a large operation and overhaul system, so as to achieve the regulation and control integration and dispatching integration at all levels. In recent years, the frequent occurrence of large-scale power outages occurred in the world, China suffered a rare ice disaster and earthquake, and power facilities were severely damaged, but the disaster recovery dispatch center at the time lack of disaster long-distance spare capacity [1], the development of standby dispatching technology is extremely urgent.

The literature [2-8] has an important reference to this article. In this paper, we propose a distributed data acquisition system based on the analysis of the existing centralized data acquisition mode to meet the needs of dispatching integration and standby dispatching, discussed the key technologies of CIM/E file management, data synchronization and so on under the framework.

\section{Function overview}

\section{Centralized data acquisition system}

In the traditional centralized data acquisition mode, each dispatching center has an independent data acquisition system, each system maintained and operated independently. As shown in figure 1, Dispatch center 1 and 2 installed and deployed the data acquisition system 1 and 2 respectively, both of station A and station B have channel conditions which has access to the two sets of data acquisition system at the same time, station $C$ only has access to the channels of data acquisition system 1 , station $\mathrm{D}$ only has access to the channels of data acquisition system 2. Suppose the two dispatching centers need to monitor all four plant stations, then the two sets of data acquisition system must collect all four station data respectively. To this end, for station A and station B whose communication link is complete, we adopt the way of redundant collection, for station $\mathrm{C}$ and station $\mathrm{D}$ which lacks the communication link, we adopt the way of data exchange between dispatching centers. 


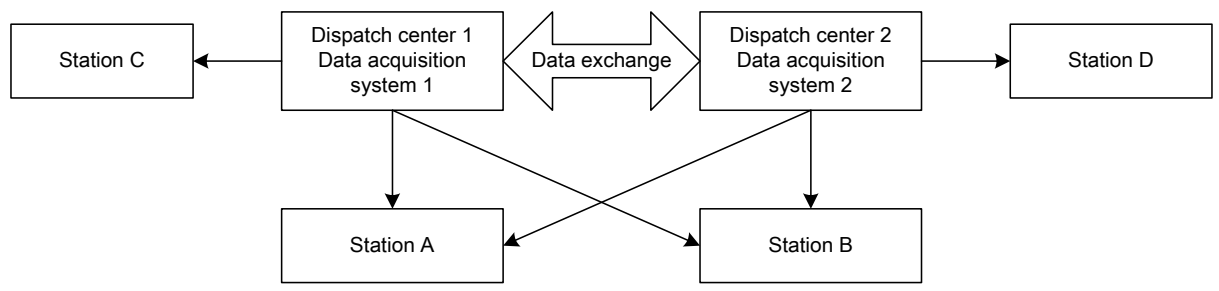

Fig. 1 Centralized data acquisition mode

It can be seen from this example, the main problems of the centralized data acquisition system in the collaborative operation are: (1)Channel redundancy construction, it is need to build a number of channels between the plant station and multiple dispatch centers.(2)Repeated maintenance, multiple dispatch centers need to do the work of link debug and parameter maintenance repeatedly for the same plant station, for individual plant station also need to increase the data exchange link.

\section{Distributed data acquisition system}

The distributed data acquisition system can be divided into multiple data acquisition subsystems according to different needs of users. Different subsystems run cooperatively, work together to complete data acquisition tasks. Still use the application scenarios of 1.1, If using distributed data acquisition system, firstly, the data acquisition subsystem 1 and 2 are deployed in the two dispatching centers. Secondly, divide the tasks, Station A and C access to data acquisition subsystem 1, Station Band $\mathrm{D}$ access to data acquisition subsystem 2. The distributed data acquisition system is shown in figure2.

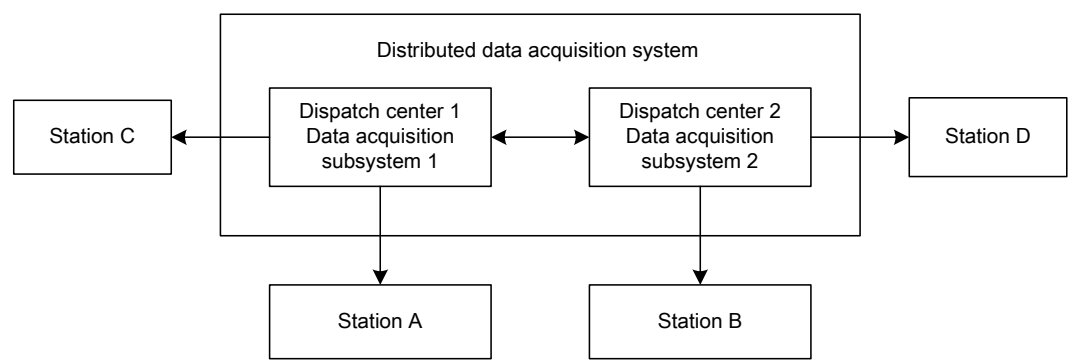

Fig. 2 distributed data acquisition system

In any one of the dispatching center we can monitor the real-time data of all four plant stations, and do not need to care about how the specific acquisition task is assigned. Real-time data which can access to any data acquisition subsystem can be shared to the whole distributed data acquisition system.The process is automatically realized by software, no human intervention, no need to establish a data exchange link. Distributed data acquisition system is suitable for data acquisition application scenario under cooperative operation mode, such as coordinated operation of province and region integration, the integration of cities and counties, main/standby integration, it can effectively solve the problems of the traditional centralized data acquisition system.

\section{Key technology research}

\section{Overall architecture}

This paper studies the distributed acquisition system using a service-oriented architecture, from the bottom up, including data acquisition layer, data storage layer, proxy service layer, maintenance and presentation layer. The data acquisition layer can achieve the conventional data acquisition function, Responsible for communication with field equipment and protocol processing; Data storage layer provides the parameter storage based on CIM/E file and real-time data storage based on shared memory; The proxy service layer includes data service, file service and monitoring service, as a connection link between the layers and the subsystems, Real time data sharing, CIM/E file management and resource agent are realized; maintenance display layer includes operation monitoring and parameter maintenance interface, providing efficient and easy-to-use man-machine interface. The overall architecture of the distributed data acquisition system is shown in figure 3. 


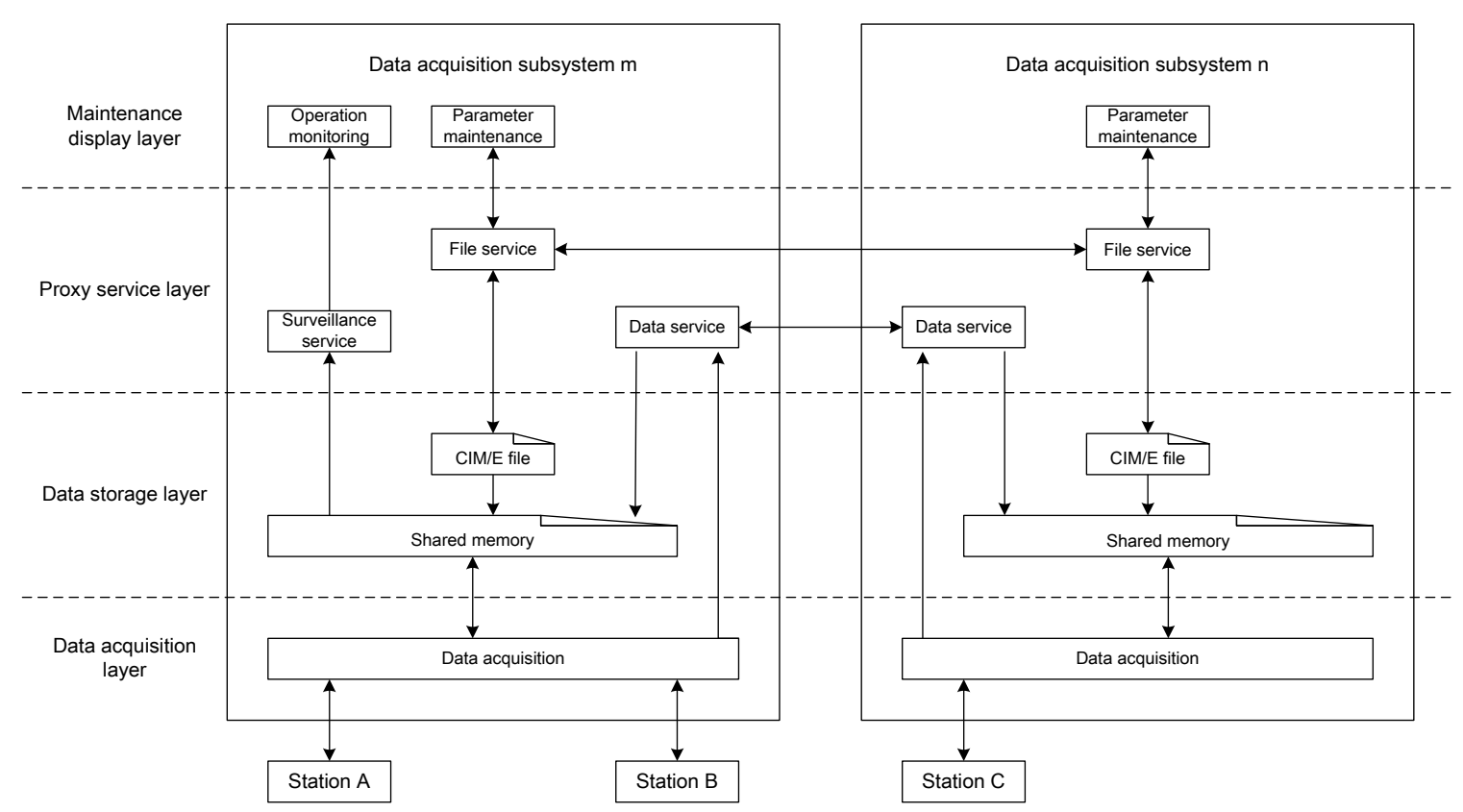

Fig. 3 Architecture of distributed data acquisition system

\section{CIM/E file management}

\section{1) $\mathrm{CIM} / \mathrm{E}$ file description}

CIME file can be divide into three categories by the different uses of the parameters in the file. The First is used to store field devices parameters and communication links parameters .The second is used to store the variable definition of protocol. Each kind protocols are saved in different files named by the protocol's name. The third is used to store index tables. Every substation has a file named by the substation name .This file stores the remote sign, remote measure, remote control, remote adjustment data of the substation. The classification of the CIM/E file is shown in Table 1.

Table 1 classification and description of CIM/E file

\begin{tabular}{ccc}
\hline Classification & CIM/E file name & File content \\
\hline Class I & link_conf.CIME & Field equipment and \\
& & communication link parameters \\
Class II & iec104_info.CIME and so on & Specification for variable parts \\
Class III & XXXstation.CIME and so on & Bilateral index table \\
\hline
\end{tabular}

Every subsystem of the data acquisition system is maintained separately and has different data acquisition assignment, so there are different CIM/E files. The subsystem could browse or modify other subsystem's file .but the file can be reused in other subsystem .In one subsystem, the file on each node are same .On Each node there are two kinds of CIM/E files, one is called modify files the other is called publish files .the modify can be made in the modify files, the publish files is used to publish the result.

2) The principle of CIM/E files access and storage

During the system maintain period, the user will browse parameters, modify and verify them .These parameters stored in CIM/E files .So we must develop the effective and reused file access interfaces .The interfaces includes common interfaces and verify interfaces .The common interfaces is used to create, read, update and delete the CIM/E files, so the file can be used like database. The verify interfaces can verify the context of the file by the pre-set principles, generate statistics and transform the subsystem the data points ID.

During the system running period, the application of distributed the data acquisition system will access the parameters frequently. The common interface can not meet the demand, so we must develop a fast storage and index technology. 


\section{3) Power grid equipment unified nomenclature}

Each dispatch centers often take independent definition the local power grid model management methods. The same equipment in different dispatch center may have different naming, has a different ID in the database. This results in data acquisition subsystem does not recognize the real-time data between each other, a real-time data acquisition subsystem can not be used by other subsystems. For this purpose each dispatch center must use the same naming grid equipment.

\section{Data Sharing}

The real time data acquired by any subsystem can be shared in the whole distributed data acquisition system. In the system maintenance phase, a number of monitoring areas for each station can be defined. The monitoring area limits the sharing range of real time data of station. Figure 4 as an example, station A is collected by data acquisition subsystem 1, the monitoring area of station A includes data acquisition subsystem 1 , subsystem 2 , subsystem 3 , thus data acquisition subsystem 1 receives the real time data of the station A automatically transfer to the data acquisition subsystem 2 and subsystem 3. Due to the monitoring area of the plant station A does not include data acquisition subsystem 4, so the station A real time data will not be released to subsystem 4.

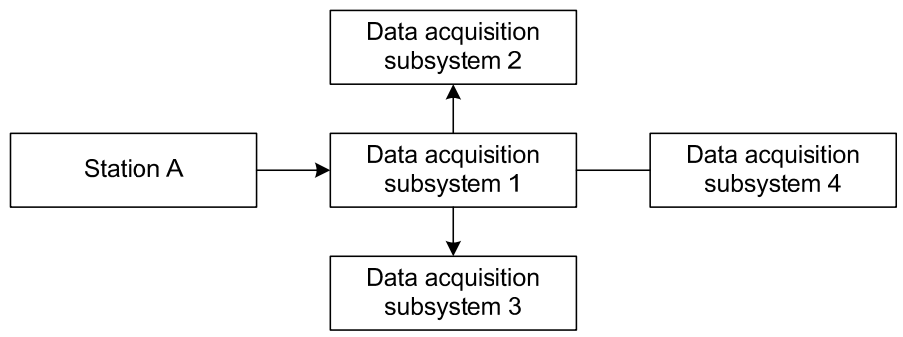

Fig. 4 data sharing of distributed data acquisition system

Specifically, data acquisition subsystem 1 will transfer real time data of station A to to the data acquisition subsystem 2 and data acquisition subsystem 3 by data services. Each real time data transmission structure includes subsystem number, station number, index number, data value, quality stamp and time stamp. After receiving the data from the data receiver, firstly the look-up table of the shared memory is queried, and the subsystem number and the station number are converted to the local station number. And then, according to the local station number and index query sharing memory, access to the real time data in the sharing memory of the corresponding local storage. Writes the real data value, quality stamp and the time stamp to the local storage record, and obtains the local data corresponding to the real time data. Finally, the local data ID, data value, quality stamp and time stamp will be transfered to the local SCADA application.

\section{Engineering test}

The distributed data acquisition system developed in this paper has been put into operation in State Grid Jibei Electric Power Company Limited and passed site acceptance test (SAT). In Jibei main dispatching center, Jibei standby dispatching center, Langfang dispatching center, Chengde dispatching center were deployed a set of data acquisition subsystem respectively, thus forming the integration of province and region, main and standby for the integration of the data acquisition mode. Within the scope of whole system access station more than 150 seats, including Jibei main dispatching center access station 52 seats, Jibei standby dispatching center access station 52 seats, Langfang dispatching center access station 48 seats, Chengde dispatching center access station 49 seats. According to the actual work needs, Langfang dispatching center and Chengde dispatching center access station 97 seats all sharing to Jibei main dispatching center and Jibei standby dispatching center. In Jibei main dispatching center and Jibei standby dispatching center both can monitor to the local access station and remote access station, the data acquisition subsystem of Jibei main dispatching center simulated fault, the data acquisition of Jibei standby dispatching center is not affected during the fault,it can bear data acquisition and monitoring tasks. Distributed data acquisition system based on CIM/E in State Grid Jibei Electric Power Company Limited, after put 
into operation, has realized the tone at the province and region, main and standby, preparation and process of the integration of maintenance, management and data sharing.

\section{Conclusion}

In this paper, the limitation of centralized data acquisition system in cooperative operation mode of multi - dispatching center is analyzed, The characteristics of distributed data acquisition system is introduced, a distributed data acquisition system based on CIM/E is proposed to meet the requirements of integration of main dispatching and standby dispatching, introduces the system architecture, discusses its key technologies, and gives the engineering test sample. Engineering test show that the performance and efficiency of CIM/E can be used to solve the problem of parameter transfer and reuse between the upper level and lower level d, the main and standby dispatching center, the system can meet the requirements, of integrative dispatch center-control center and standby dispatching center.

\section{Acknowledgements}

Science and technology projects of State Grid Corporation of China "Research and application of key technology optimization of dispatching technology support system oriented large operation and overhaul”

\section{Reference}

[1] XIN Yaozhong, SHI Junjie, ZHOU Jingyang, et al. Technology development trends of smart grid dispatching and control systems [J]. Automation of Electric Power Systems, 2015, 39(1): 2-8.

[2] CHEN Ning, XU Chunlei, ZHUANG Weijin, et al. Distributed data acquisition of unified and county dispatching automation system [J]. Automation of Electric Power Systems, 2011, 35(24): 89-92.

[3] XIN Yaozhong, MI Weimin, JIANG Guodong, et al. Scheme of application model information sharing between control centers Based on CIM/E [J]. Automation of Electric Power Systems, 2013, 37(8): 1-5.

[4] LIU Tao, MI Weimin, CHEN Zhengping, et al. Integrated sharing scheme for grid model and graphics applicable to grand operation system [J]. Automation of Electric Power Systems, 2015, 39(1): 36-41.

[5] CHANG Guanghui, HAO Junjie, LIU Baojiang, et al. Research and development of intelligent and classified collection system for electric power dispatching and control informateion[J]. Power System Protection and Control, 2015, 43(6):115-120.

[6] ZHAO Jiaqing, ZHAO Yuxiao, DING Hongen, et al. Technical scheme for the verification of grid models between the active and standby systems in power dispatching automation system [J]. Power System Protection and Control, 2014, 42(19):139-144.

[7] ZHU Botong, CHENG Zhihai, TANG Zhiqiang, et al. Interoperate research of the intelligent substation and dispatching center based on CIM model [J]. Power System Protection and Control, 2013, 41(10):93-97.

[8] TIAN Jiang, QIAN Kejun, LIANG Feng, Application of distributed state estimation in smart substation [J]. Power System and Clean Energy, 2013, 29(9):27-31. 